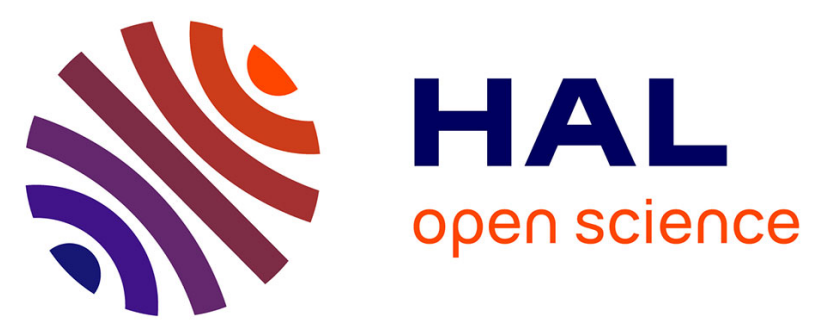

\title{
Effect of the nitrogen incorporation and fast carrier dynamics in (In,Ga)AsN/GaP self-assembled quantum dots
}

Jean-Philippe Gauthier, Cédric Robert Robert, Samy Almosni, Yoan Léger, Mathieu Perrin, Jacky Even, Andrea Balocchi, Hélène Carrère, Xavier Marie, Charles Cornet, et al.

\section{To cite this version:}

Jean-Philippe Gauthier, Cédric Robert Robert, Samy Almosni, Yoan Léger, Mathieu Perrin, et al.. Effect of the nitrogen incorporation and fast carrier dynamics in (In,Ga)AsN/GaP self-assembled quantum dots. Applied Physics Letters, 2014, 105 (24), pp.243111. 10.1063/1.4904939 . hal-01114385

\author{
HAL Id: hal-01114385 \\ https://hal.science/hal-01114385
}

Submitted on 9 Feb 2015

HAL is a multi-disciplinary open access archive for the deposit and dissemination of scientific research documents, whether they are published or not. The documents may come from teaching and research institutions in France or abroad, or from public or private research centers.
L'archive ouverte pluridisciplinaire HAL, est destinée au dépôt et à la diffusion de documents scientifiques de niveau recherche, publiés ou non, émanant des établissements d'enseignement et de recherche français ou étrangers, des laboratoires publics ou privés. 


\title{
Effect of the nitrogen incorporation and fast carrier dynamics in (In,Ga)AsN/GaP self-assembled quantum dots
}

\author{
J.-P. Gauthier, ${ }^{1}$ C. Robert, ${ }^{2}$ S. Almosni, ${ }^{1}$ Y. Léger, ${ }^{1}$ M. Perrin,,${ }^{1}$ J. Even,,${ }^{1}$ A. Balocchi, ${ }^{3}$ H. \\ Carrere, ${ }^{3}$ X. Marie, ${ }^{3}$ C. Cornet,,${ }^{1, *}$ and O. Durand ${ }^{1}$ \\ ${ }^{1}$ UMR FOTON, CNRS, INSA-Rennes, F-35708, Rennes, France \\ ${ }^{2}$ Tyndall National Institute, University College Cork, Lee Maltings, Cork, Ireland. \\ ${ }^{3}$ Université de Toulouse, INSA-CNRS-UPS, LPCNO, F- 31077 Toulouse, France
}

\begin{abstract}
We report on the structural and optical properties of (In,Ga)AsN self-assembled quantum dots grown on GaP (001) substrate. A comparison with nitrogen free (In,Ga)As system is presented, showing a clear modification of growth mechanisms and a significant shift of the photoluminescence spectrum. Low temperature carrier recombination dynamics is studied by time-resolved photoluminescence, highlighting a drastic reduction of the characteristic decaytime when nitrogen is incorporated in the quantum dots. Room temperature photoluminescence is observed at $840 \mathrm{~nm}$. These results reveal the potential of ( $\mathrm{In}, \mathrm{Ga}) \mathrm{AsN}$ as an efficient active medium monolithically integrated on Si for laser applications.
\end{abstract}

Keywords: photonics on silicon, InGaAsN/GaP, quantum dots, room temperature photoluminescence, carrier dynamics, dilute nitride.

P.A.C.S.: 78.67.De - 78.67.Hc-68.65.Hb-71.15.Dx-81.07.Ta-78.47.Jd 
The integration of optoelectronic laser devices on silicon $(\mathrm{Si})$ is one of the major contemporary challenges for optoelectronic circuits and optical interconnects. To this aim, the pseudomorphic growth of III-V semiconductor materials on $\mathrm{Si}$ was recently proposed to overcome the issue of misfit dislocations which presently limits the performances of metamorphic III-V lasers monolithically integrated on silicon. Among all III-V materials, gallium phosphide $(\mathrm{GaP})$ is a very promising candidate, as its lattice constant is the closest to $\mathrm{Si}(0.37 \%$ lattice mismatch at $300 \mathrm{~K})$. Despite its intrinsic strucural benefits, $\mathrm{GaP}$ presents an indirect bandgap $(2.3 \mathrm{eV})$, which, at first glance, is detrimental for the fabrication of highly efficient semiconductor laser diodes. In this materials system, the use of GaAsPN quantum wells as a gain medium for lasing was first proposed and an electrically pumped laser on silicon was demonstrated under pulsed operation below $150 \mathrm{~K} .{ }^{1}$ In this structure, the quantum efficiency of the active area faces however the intrinsic limitation of the pseudo-direct bandgap of the GaAsPN alloy, where nitrogen localized states are located below the conduction band minimum, and the strained GaAs on GaP/Si is of indirect type. ${ }^{2}$ More recently, high (In,Ga)As quantum dots densities on GaP substrate were proposed as a credible alternative for lasing on $\mathrm{GaP} / \mathrm{Si}^{3}{ }^{3}$ With these materials, lasing was achieved on $\mathrm{GaP}$ substrate at $80 \mathrm{~K}$ under pulsed operation. ${ }^{4} \mathrm{~A}$ light-emitting diode was even demonstrated on the GaP/Si pseudo-substrate. ${ }^{5}$ However, the excellent structural properties of $(\mathrm{In}, \mathrm{Ga}) \mathrm{As} / \mathrm{GaP}$ quantum $\operatorname{dots}^{6,7}$ are balanced by the $\Gamma$-X competition at the conduction band minimum, confirmed both theoretically ${ }^{8}$ and experimentally ${ }^{9}$, and leading to a long radiative decay time for the ground state transition. ${ }^{8,10}$ The indirect to direct cross-over was finally demonstrated very recently on GaP substrate playing on the QD shape and strain relief, thus demonstrating the potential achievement of a true direct bandgap optical transitions on $\mathrm{Si}$ substrate in the pseudomorphic approach at $750 \mathrm{~nm}^{11}$ In this context, incorporation of nitrogen in (In,Ga)As QDs may provide crucial advantages, due to the strong band-gap bowing effect, which could allow extending the emission wavelength to the datacom/telecom range, and a shift of the direct transition to lower energies than indirect ones. Indeed, the use of (In,Ga)AsN active areas on GaAs was studied intensively several years ago, and promising devices performances were obtained. ${ }^{12}$ In these materials systems, the ground state transition has significant quantum efficiency, because localized nitrogen states are located above the conduction band minimum at the $\Gamma$ point. Pioneering experiments on (In,Ga)AsN/GaP QDs were performed by Umeno et al. in 2010, where the first low intensity room temperature Photoluminescence (PL) was demonstrated on a 5-stacked QDs sample. ${ }^{13}$ In 2011, Urakami et al. proposed to use a Rapid Thermal Annealing (RTA) procedure to increase the structural quality and enhance the PL efficiency for the QDs. ${ }^{14}$ Finally, Fukami et al. predicted a direct bandgap optical transition up to $1100 \mathrm{~nm}$ using a $50 \%$ In composition and an $\mathrm{N}$ 
composition between 1 and $2 \%$ with the Band Anti-Crossing model. ${ }^{15}$ In these works, the QDs photoluminescence efficiency comparison with nitrogen-free (In,Ga)As/GaP QDs was not adressed. Furthermore, the experimental determination of carrier dynamics on the different QDs energy levels was not measured, which is a crucial information for further device developments.

In this letter, we report on the time-resolved optical properties of self-assembled InGaAsN quantum dots, grown by molecular beam epitaxy and compare their properties to a $\mathrm{N}$-free InGaAs QD system. We first compare structural properties of QDs with or without nitrogen by Atomic Force Microscopy (AFM). We then study the effect of the incorporation of nitrogen in InGaAs/GaP QDs on the (time-resolved) photoluminescence at room and low temperatures. We finally discuss the influence of band structure, QDs inhomogeneity and non-radiative relaxation channels on the shorter decay times measured for (In, Ga)AsN QDs.

Four InGaAs(N) QDs samples were grown on an unintentionally doped GaP(001) substrate using a Solid-Source Molecular Beam Epitaxy apparatus. ${ }^{16}$ Two samples are composed of nitrogen-free single plane of QDs having exactly the same growth conditions, but with or without a $30 \mathrm{~nm}$-thick GaP capping layer for PL and AFM analysis respectively. The two other samples are composed of (In,Ga)AsN/GaP QDs, with or without GaP capping layer, for the same kind of analysis. For all the samples, the nominal (In,Ga)As thickness deposited was 3ML and the nominal composition of In was set to $30 \%$ (the In composition is not homogeneous within the QDs). ${ }^{7,8}$ For nitrogen-free (In,Ga)As/GaP QDs samples, growth conditions are described in detail in previous works. ${ }^{3,8}$ In the case of (In,Ga)AsN QDs, growth conditions are exactly similar to their nitrogen-free counterpart, except that growth was performed at slightly lower temperature $\left(540^{\circ} \mathrm{C}\right.$ instead of $580^{\circ} \mathrm{C}$ for the nitrogen-free structure, as measured by an optical pyrometer) to avoid desorption of nitrogen atoms and control the nitrogen incorporation. ${ }^{17}$ In this case, the valved nitrogen RF plasma source is switched on during the 3ML deposition of (In, Ga)As to incorporate nitrogen in the nanostructures. $\mathrm{RF}$ power is set to $400 \mathrm{~W}$ and $\mathrm{N}_{2}$ flow to 0.5 sccm. Nominal composition of indium was set to $30 \%$ in the four samples. In (In,Ga)AsN QDs, the targeted nitrogen composition is $1.5 \%$, assuming the same incorporation processes as for GaPN growth. ${ }^{17}$ RTA post-growth treatments were performed on (In,Ga)AsN QDs during one minute at $800^{\circ} \mathrm{C}$ under forming gas flow $\left(\mathrm{H}_{2}+\mathrm{N}_{2}\right)$. Without any post growth thermal treatment, room temperature photoluminescence was not observed for the (In,Ga)AsN QDs sample. Contact mode AFM was used to determine the QDs sizes and density. Continuous wave (cw) PL experiments were performed in a close-cycle Helium bath cryostat using an excitation laser emitting at $480 \mathrm{~nm}$ (power density $\sim 20 \mathrm{~W} . \mathrm{cm}^{-2}$ ). Time-resolved PL experiments were performed at low temperature exciting the sample with a 1 ps pulse-width frequency-doubled Ti:Sapphire laser at $405 \mathrm{~nm}$ with a repetition frequency of 80 
MHZ. The PL intensity was then dispersed by an imaging spectrometer. The signal dynamic was detected by an S1-photocathode streak-camera with an overall temporal resolution of 8 ps.

A comparison of the structural properties for QDs with or without nitrogen based on 1x1 $\mu \mathrm{m}^{2} \mathrm{AFM}$ images, represented in Fig. 1(a) for (In,Ga)As QDs and in Fig. 1(b) for (In,Ga)AsN QDs, is summarized in Fig. 1(c). A clear reduction of the QD density (from $1.7 .10^{11} \mathrm{~cm}^{-2}$ to $3.10^{10} \mathrm{~cm}^{-2}$ ) is first observed for nitrogen containing samples, together with an increase of heights and radii, as compared to nitrogen-free QDs samples, despite the same material quantity deposited in both cases. This highlights the predominant role of nitrogen atoms during the QDs nucleation, and may entail a significant incorporation of nitrogen inside quantum dots. Such a difference is indeed attributed to a reduced lattice-mismatch with the GaP substrate, i.e. the compressive strain is significantly reduced in the QDs with the incorporation of nitrogen. Note that in both cases, no bimodal distribution was observed by AFM, contrary to the works of Heidemann et al. ${ }^{4}$ Both InGaAs $^{6}$ and InGaAsN QD exhibit a clear correlation between height and radius, with a broader statistical distribution in the case of InGaAsN QD. For nitrogen containing QDs, the height $\frac{\Delta h}{h}$ and radius $\frac{\Delta r}{r}$ dispersions for uncovered QD, are respectively $31 \%$ and 35\%, slightly larger than other InAs-based QD systems but still in a reasonable range (see ref. [3] and references therein).

Figure 2 shows the low $(20 \mathrm{~K})$ and room temperature photoluminescence (PL) of InGaAs (red line) and InGaAsN QDs (blue line). A strong redshift of the PL peak is first observed when adding nitrogen, from $1.77 \mathrm{eV}$ $(700 \mathrm{~nm})$ to $1.48 \mathrm{eV}(837 \mathrm{~nm})$ at room temperature. The difference in QDs size between the two QDs systems, even if it is significant, cannot fully explain such a large energy shift. ${ }^{8}$ This is more likely explained by the giant bandgap bowing usually observed in dilute nitrides systems, where the localized nitrogen states couple to the conduction band. ${ }^{2}$ This is consistent with effective nitrogen incorporation inside the QDs. The stronger quenching of the PL with increasing temperature (factors of 2 and 30 for InGaAs and InGaAsN QDs respectively as shown in Fig. 2), may be attributed to different band lineups, strain status, or quantum confinement. ${ }^{15}$ But this could also be explained by the appearance of structural defects leading to non-radiative recombinations when nitrogen is introduced. In the case of nitrogen-free (In,Ga)As QDs, a double peak structure is observed at low temperature. A low energy transition (LE) at $1.76 \mathrm{eV}$ and a higher energy transition (HE) at $1.86 \mathrm{eV}$ are observed. This has been already widely discussed in previous works and attributed to both the $\mathrm{GaP} \mathrm{X}_{\mathrm{z}}$ conduction band states for the lower energy transition, and to the direct $\Gamma$ conduction band states for the higher energy transition. ${ }^{9}$ Slow (LE) and fast (HE) recombination dynamics have been measured by TRPL. ${ }^{8}$ The Full Width at Half Maximum (FWHM) of the 
two transitions is measured to be around $100 \mathrm{meV}$, with a separation between both transition being about 100 meV. ${ }^{8}$ In this case, the higher energy transition is activated by increasing the PL incident laser power or the sample temperature.

For (In,Ga)AsN QDs, the situation is radically different, as the higher energy transition mainly contributes at low temperature, and is nearly not visible at room temperature. This tends to exclude the contribution of conduction band excited states located at different point of the Brillouin zone like in InGaAs QD. Figure 3 presents the temperature dependence of the cw-PL spectra obtained on (In,Ga)AsN QDs between $20 \mathrm{~K}$ and room temperature (RT). Indeed, the HE transition (1.63eV) rises when decreasing temperature while the LE transition is the main PL contribution at room temperature. A double Gaussian fit is used to determine the properties of these transitions as a function of temperature. The FWHM of both peaks is evaluated to be around $125 \mathrm{meV}$ through the fitting of the peaks at low temperature on the high energy side. The low energy tail (LET), observed down to $1.2 \mathrm{eV}$, is commonly attributed to Nitrogen-induced localized states through alloy fluctuations in dilute nitrides systems, ${ }^{18}$ and therefore is not considered in the fitting procedure. The separation between LE and HE transitions is in the [110-150] meV range. These observations indicate that the two transitions observed in this case may come, from QD and Wetting Layer (WL) contributions, or from a QD bimodal distribution. Inset of Fig. 3 represents the evolution of the integrated PL intensity as a function of the inverse temperature, as determined by previous multiple peak fitting for HE and LE transitions. Curves are fitted using an Arrhenius law with two activation energies. For both transitions, activation energies are found to be in the same order of magnitude. Inversion of slope is not observed on these curves. Therefore no evidence of carrier redistribution can be seen between the two involved states, confirming the independence of the optical transitions, even at room temperature. ${ }^{19}$ A first activation energy is found to be a few meV, and a second one a few tens of meV. This reflects a similarity between the quantum states involved in the recombination process, which tends to favor the hypothesis of a QD bimodal distribution. Note that even if AFM analysis did not allow identifying bimodal size distribution, a bimodal distribution could be created during the GaP capping, or the RTA process. This feature was already observed for InAsN/GaAs QDs. ${ }^{20}$ Moreover, a different incorporation of nitrogen atoms inside the QDs could possibly create a bimodal composition distribution, i.e. QDs having the same size, but different optical properties due to the different configurations of nitrogen incorporation inside the QD.

The carrier dynamics has been studied by Time-Resolved PL (TRPL) experiments. Figure 4-(a) and (b) present the streak camera images measured for (In,Ga)AsN and (In,Ga)As QDs at a temperature of 8K, under a timeaveraged excitation power of $22 \mathrm{~mW}$. A first observation of these traces obviously demonstrates a much smaller 
decay time for QDs including nitrogen. The PL intensity is plotted in Fig. 4(c) as a function of time at selected energies: the low energy peak for (In,Ga)As QDs and LE, HE and LET for (In,Ga)AsN QDs. The PL intensities have been corrected to take into account the wavelength dependence of the detector sensitivity and the relative gain used to maximize detection. Decay times are extracted from the long constant decay of a biexponential fit. As shown in previous work, (In,Ga)As QDs present a long decay time $(>1 \mu \mathrm{s})$, related to the indirect character of the LE transition. ${ }^{8}$ On the opposite, the PL dynamics of (In,Ga)AsN QDs is much faster. A multiexponential fast decay (a few hundreds of ps) is first observed, which reveals the influence of non-radiative Auger processes in the dots. ${ }^{21}$ After about 500 ps, a mono-exponential decay is restored, still much shorter than for nitrogen-free QDs. Decay times of 700 ps, $1 \mathrm{~ns}$, and $1.3 \mathrm{~ns}$ are extracted for LET, LE, and HE transitions respectively. Note that these decay times are of the same order of magnitude as those measured in InAsN/GaAs QDs. ${ }^{22}$ The PL rise-time is in the range of a few tens of ps at all energies. The similar behaviors of the LE and HE peaks indicate that there is no population exchange between the two transitions, confirming the previous hypothesis of two classes of QDs with independent dynamics. Carriers relax independently from the barrier and wetting layer in each kind of dots and no spatial redistribution occurs due to the low temperature of the experiment. In this context, the short decay time might be attributed to fast non-radiative processes, which may come from structural defects due to the nonoptimized growth conditions. With this assumption, the activation energies extracted from Arrhenius plots of Fig. 3 inset reflects the energy needed to reach excited states with low optical efficiency, such as non-radiative centers. Further electronic structure calculations will be needed to clarify this point in such a complex system.

In summary, this paper presents a comprehensive comparison of structural, optical and carrier dynamics properties of $(\mathrm{In}, \mathrm{Ga}) \mathrm{As} / \mathrm{GaP}$ and $(\mathrm{In}, \mathrm{Ga}) \mathrm{AsN} / \mathrm{GaP}$ QDs. It is shown that nitrogen strongly impacts the QD nucleation. A strong red-shift of the photoluminescence with nitrogen incorporation is also demonstrated at 840 $\mathrm{nm}$, close to the datacom applications window, together with the presence of two optical transitions in the (In,Ga)AsN system. The origin of these two transitions is found to be drastically different from what is being observed on nitrogen-free structures. Carrier dynamics is finally investigated trough TRPL, and decay times are found at low temperature to be much shorter when nitrogen is used for both transitions. The two transitions are finally attributed to bimodal and independent QD distributions, while the carrier dynamics is found to be limited by non-radiative processes. In conclusion, provided that an improvement of the QDs structural quality is achieved, these QDs may offer opportunities to push emission wavelength of direct bandgap emitters pseudomorphically integrated on silicon toward the near infrared range. 
This research was supported by "Région Bretagne" through the PONANT project including FEDER funds. This work was also supported by the OPTOSI ANR Project No. 12-BS03-002-02. 
Figure 1
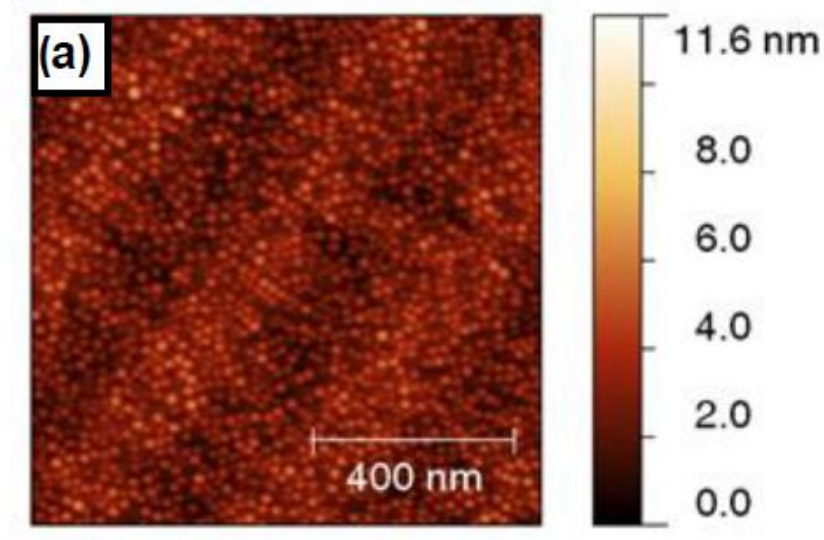

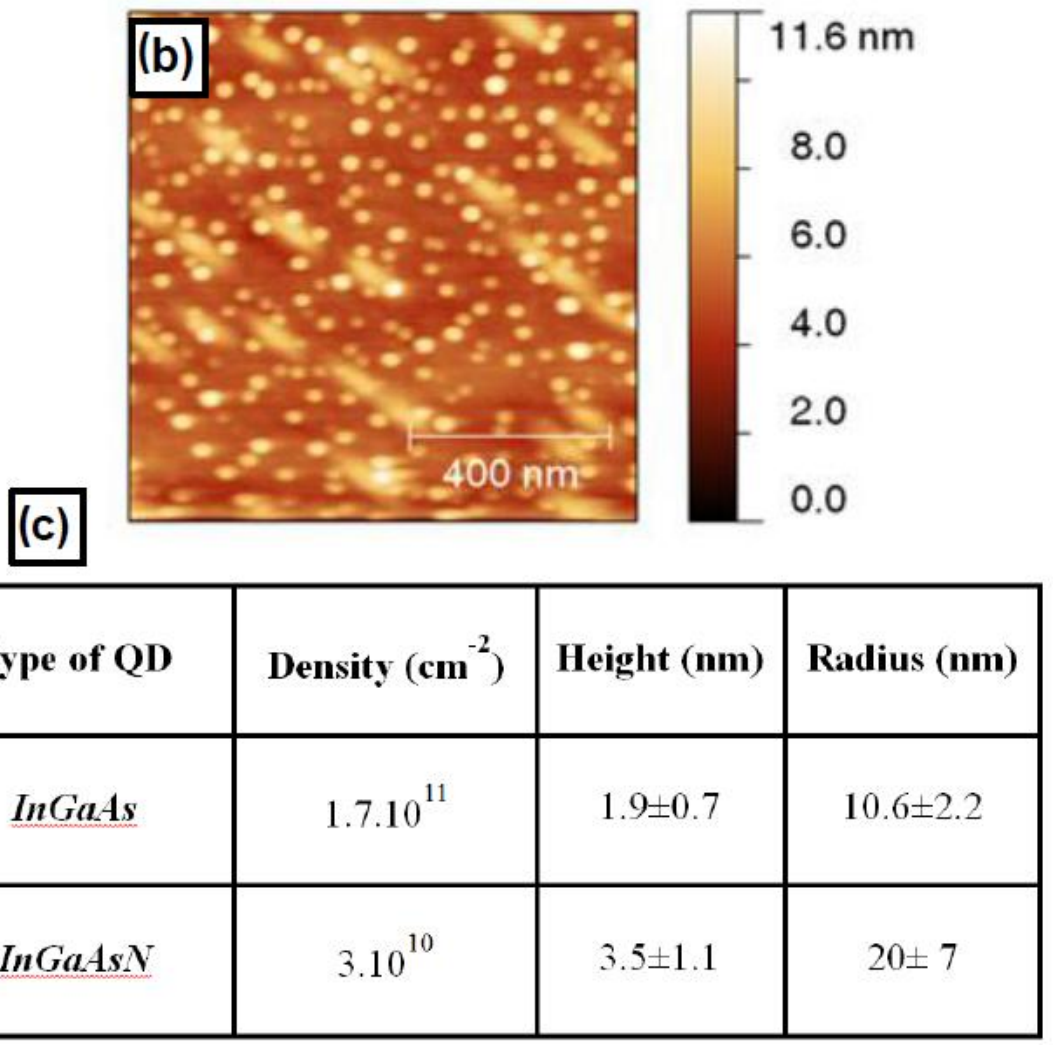


Figure 2

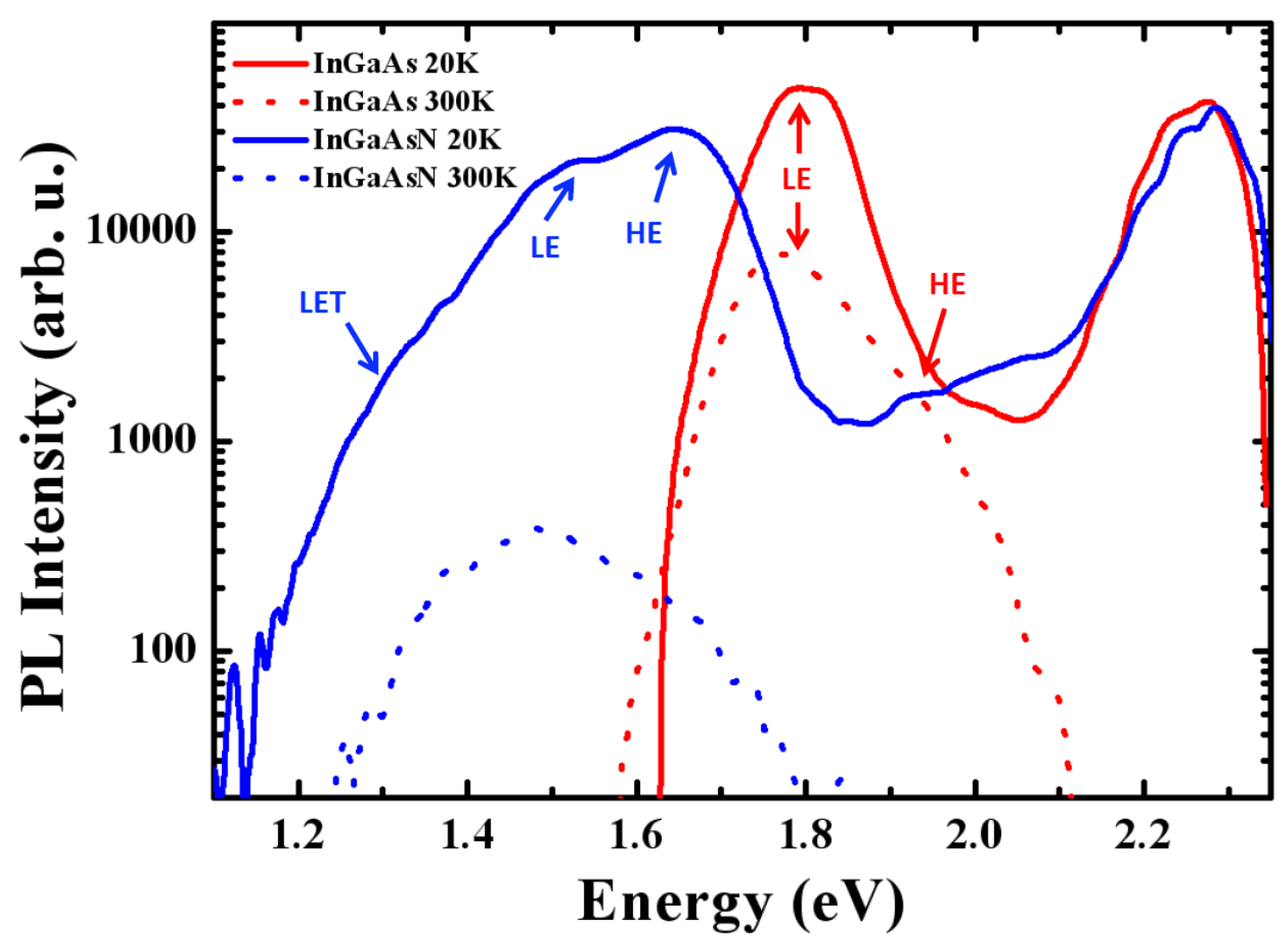


Figure 3

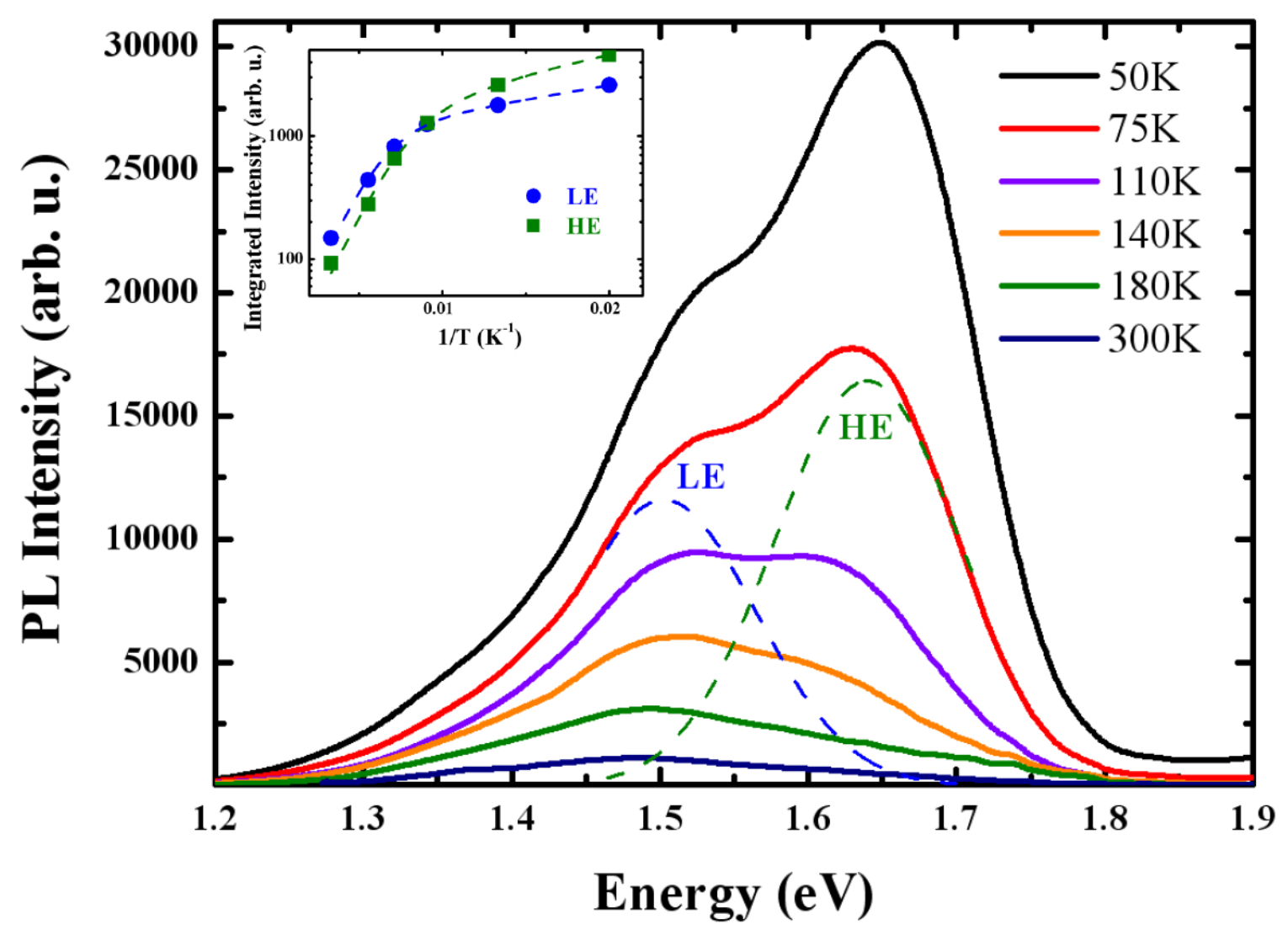


Figure 4

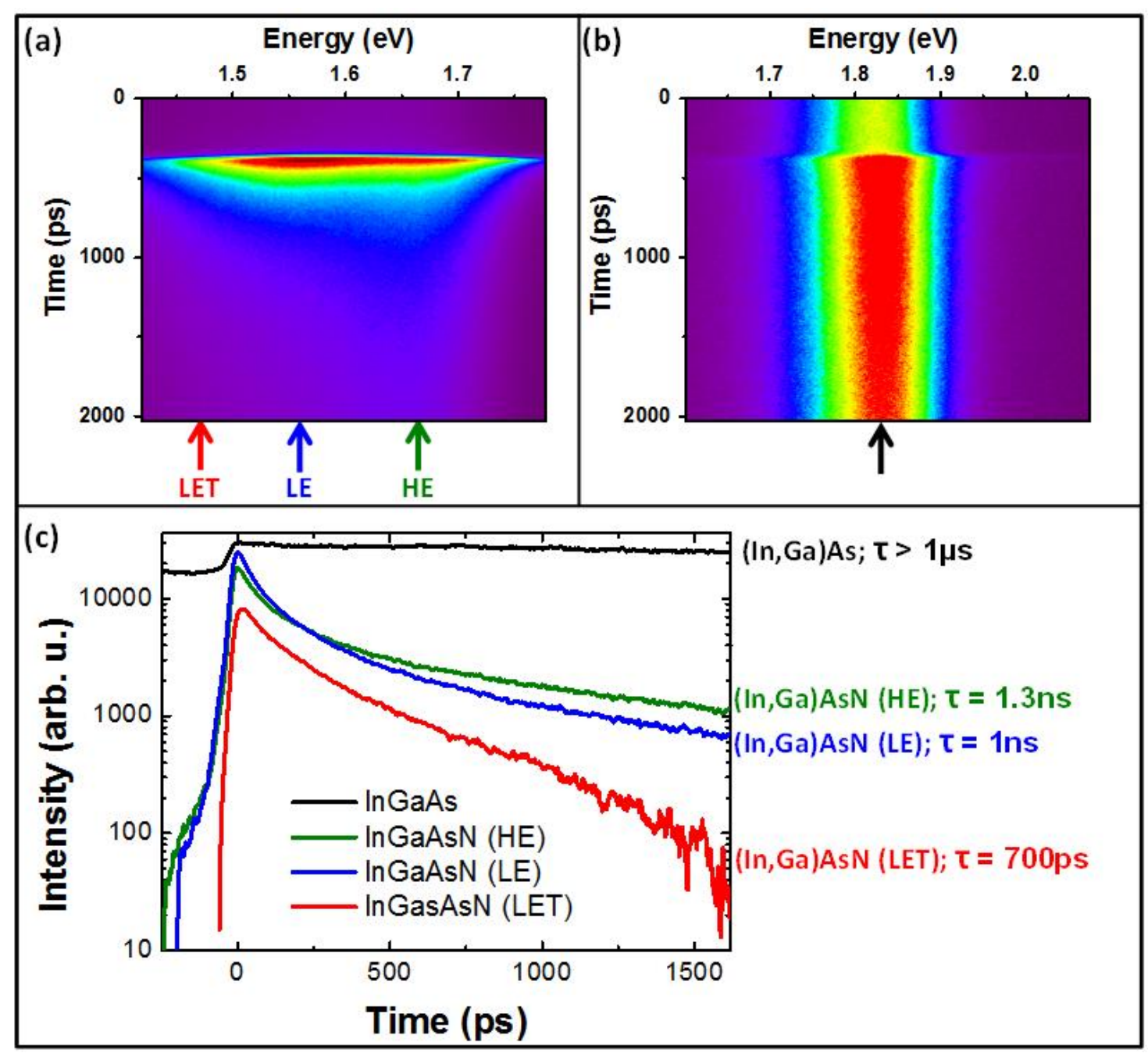




\section{FIGURE CAPTIONS}

FIG 1. $1 \mathrm{x} 1 \mu \mathrm{m}^{2}$ atomic force microscopy image from InGaAs (a) and InGaAsN (b) QDs grown on GaP. (c) Density, height, and radius of QDs with associated dispersion.

FIG. 2. Continuous-wave photoluminescence spectra of InGaAsN (blue) and InGaAs QDs (red) at $20 \mathrm{~K}$ (solid line) and room temperature (dots). Low temperature photoluminescence at about $2.3 \mathrm{eV}$ is due to the $\mathrm{GaP}$ substrate.

FIG. 3. Temperature dependent photoluminescence of (In,Ga)AsN quantum dots. Dashed lines are the two Gaussian fits extracted from the spectrum. The inset plots the integrated intensity of both fits as a function of the inverse temperature. The activation energies calculated from an Arrhenius law are respectively $5 \mathrm{meV} / 60 \mathrm{meV}$ for $\mathrm{LE}$ and $10 \mathrm{meV} / 60 \mathrm{meV}$ for $\mathrm{HE}$.

FIG. 4. Time resolved photoluminescence spectra of (In,Ga)AsN QDs (a), and (In,Ga)As QDs (b) at $8 \mathrm{~K}$. Comparison of the time resolved photoluminescence profiles (c) extracted at the low energy peak for (In,Ga)As/GaP QDs (black), and at the HE peak (green) LE peak (blue), and LET tail (red) for (In,Ga)AsN QDs. 


\section{REFERENCES}

${ }^{1}$ S. Liebich, M. Zimprich, A. Beyer, C. Lange, D.J. Franzbach, S. Chatterjee, N. Hossain, S.J. Sweeney, K. Volz, B. Kunert, and W. Stolz, Appl. Phys. Lett. 99, 071109 (2011).

${ }^{2}$ C. Robert, M. Perrin, C. Cornet, J. Even, and J.M. Jancu, Appl. Phys. Lett. 100, 111901 (2012).

${ }^{3}$ T. Nguyen Thanh, C. Robert, C. Cornet, M. Perrin, J.M. Jancu, N. Bertru, J. Even, N. Chevalier, H. Folliot, O. Durand, and A. Le Corre, Appl. Phys. Lett. 99, 143123 (2011).

${ }^{4}$ M. Heidemann, S. Höfling, and M. Kamp, Appl. Phys. Lett. 104, 011113 (2014).

${ }^{5}$ Y. Song and M.L. Lee, Appl. Phys. Lett. 103, 141906 (2013).

${ }^{6}$ C. Robert, T. Thanh, C. Cornet, P. Turban, M. Perrin, A. Balocchi, H. Folliot, N. Bertru, L. Pedesseau, M. Nestoklon, J. Even, J.-M. Jancu, S. Tricot, O. Durand, X. Marie, and A.L. Corre, Nanoscale Res. Lett. 7, 643 (2012).

${ }^{7}$ C. Prohl, A. Lenz, D. Roy, J. Schuppang, G. Stracke, A. Strittmatter, U.W. Pohl, D. Bimberg, H. Eisele, and M. Dähne, Appl. Phys. Lett. 102, 123102 (2013).

${ }^{8}$ C. Robert, C. Cornet, P. Turban, T. Nguyen Thanh, M.O. Nestoklon, J. Even, J.M. Jancu, M. Perrin, H. Folliot, T. Rohel, S. Tricot, A. Balocchi, D. Lagarde, X. Marie, N. Bertru, O. Durand, and A. Le Corre, Phys. Rev. B 86, 205316 (2012).

${ }^{9}$ C. Robert, M.O. Nestoklon, K.P. da Silva, L. Pedesseau, C. Cornet, M.I. Alonso, A.R. Goñi, P. Turban, J.-M. Jancu, J. Even, and O. Durand, Appl. Phys. Lett. 104, 011908 (2014).

${ }^{10}$ C. Robert, T.N. Thanh, A. Létoublon, M. Perrin, C. Cornet, C. Levallois, J.M. Jancu, J. Even, P. Turban, A. Balocchi, X. Marie, O. Durand, and A. Le Corre, Thin Solid Films 541, 87 (2013).

${ }^{11}$ G. Stracke, E.M. Sala, S. Selve, T. Niermann, A. Schliwa, A. Strittmatter, and D. Bimberg, Appl. Phys. Lett. 104, 123107 (2014). 
${ }^{12}$ J.S. Harris, Semicond. Sci. Technol. 17, 880 (2002).

${ }^{13}$ K. Umeno, Y. Furukawa, N. Urakami, R. Noma, S. Mitsuyoshi, A. Wakahara, and H. Yonezu, Phys. E Low-Dimens. Syst. Nanostructures 42, 2772 (2010).

${ }^{14}$ N. Urakami, K. Umeno, Y. Furukawa, F. Fukami, S. Mitsuyoshi, H. Okada, H. Yonezu, and A. Wakahara, Phys. Status Solidi C 8, 263 (2011).

${ }^{15}$ F. Fukami, K. Umeno, Y. Furukawa, N. Urakami, S. Mitsuyoshi, H. Okada, H. Yonezu, and A. Wakahara, Phys. Status Solidi C 8, 322 (2011).

${ }^{16}$ T. Quinci, J. Kuyyalil, T.N. Thanh, Y.P. Wang, S. Almosni, A. Létoublon, T. Rohel, K. Tavernier, N. Chevalier, O. Dehaese, N. Boudet, J.F. Bérar, S. Loualiche, J. Even, N. Bertru, A.L. Corre, O. Durand, and C. Cornet, J. Cryst. Growth 380, 157 (2013).

${ }^{17}$ J. Kuyyalil, T. Nguyen Thanh, T. Quinci, S. Almosni, A. Létoublon, T. Rohel, N. Bertru, A. Le Corre, O. Durand, and C. Cornet, J. Cryst. Growth 377, 17 (2013).

${ }^{18}$ C. Robert, A. Bondi, T. Nguyen Thanh, J. Even, C. Cornet, O. Durand, J.P. Burin, J.M. Jancu, W. Guo, A. Létoublon, H. Folliot, S. Boyer-Richard, M. Perrin, N. Chevalier, O. Dehaese, K. Tavernier, S. Loualiche, and A. Le Corre, Appl. Phys. Lett. 98, 251110 (2011).

${ }^{19}$ C. Cornet, M. Hayne, P. Caroff, C. Levallois, L. Joulaud, E. Homeyer, C. Paranthoen, J. Even, C. Labbé, H. Folliot, V. Moshchalkov, and S. Loualiche Phys. Rev. B 74, 245315 (2006).

${ }^{20}$ O. Schumann, L. Geelhaar, H. Riechert, H. Cerva, and G. Abstreiter, J. Appl. Phys. 96, 2832 (2004).

${ }^{21}$ S. Ghosh, P. Bhattacharya, E. Stoner, J. Singh, H. Jiang, S. Nuttinck and J. Laskar, Appl. Phys. Lett. 79, 722 (2001).

${ }^{22}$ Y.D. Jang, N.J. Kim, J.S. Yim, D. Lee, S.H. Pyun, W.G. Jeong, and J.W. Jang, Appl. Phys. Lett. 88, 231907 (2006). 